\title{
BOUNDARY EFFECT BEHAVIOR IN THE HEXAGONAL-TYPE BIPERIODIC STRUCTURES WITH ROTATIONAL SYMMETRY
}

\author{
Marta Mazewska, Dorota Kula, Ewaryst Wierzbicki \\ Faculty of Civil and Environmental Engineering \\ Warsaw University of Life Sciences - SGGW, Poland \\ marta_mazewska@sggw.pl,kuladorota@wp.pl,ewarystwierzbicki@interia.pl
}

\begin{abstract}
The report presents a study of the thermal boundary effect behaviour in the hexagonal-type rigid conductors. Every hexagonal cell of the conductor is made of three rhombus parts. Some special cases of a considered conductor with rhombus parts built of two layers of different isotropic material are analyzed. The tolerance averaging approach is used as a tool of modeling. The most important result of the paper is specification of parameters which determine the character and the intensity of the boundary effect behavior. Introduced in the contribution the boundary effect intensity parameter is suggested to be treated as a certain measure of the intensity of boundary effect behavior.
\end{abstract}

Keywords: tolerance averaging, heat conduction, biperiodic conductors, boundary effect

\section{Introductory concepts}

The behavior of perturbation of the various physical fields caused by its interacting jointly with the boundaries of the regions occupied by these fields is usually referred to as the boundary effect behavior. Since the nature of boundary effect behavior is very complicated, it is difficult to obtain an exhausting description of that effect. In this contribution, considerations are restricted to descriptions of the behaviors interrelated with the boundary effects caused by the hexagonal microstructure of the conductor. The aim of the paper is to propose the averaged model of boundary effect behavior and to show that in the framework of proposed model it reduces to the suppressed expansion of the near-boundary fluctuations of the temperature field into the interior of the conductor.

The tolerance averaging is taken into account as the tool of modeling. The choice of this method seems to be natural since in this approach the thermal state of the conductor is described not by the classical temperature field but by the pair of fields: averaged temperature field (over periodicity cells) and the fluctuation amplitudes fields. For details the reader is referred to [1-4]. The biperiodic hexagonal-type material structure with rotational symmetry is illustrated in (Fig. 1). The object of interest of this paper is a special kind of behavior observed in periodic structures that is called boundary effect behavior. It consists of suppressing the 
fluctuation amplitudes in very thin selvedge layer in conductor. To describe the boundary effect there has been used the boundary effect equation, cf. [5, 6], which is part of the second of tolerance model equations obtained using the tolerance averaging technique, cf. $[2,7,8]$, from parabolic heat transfer equation. Similar methods of modeling hexagonal-type structures were also analyzed in [9-11].

For given periodic structure parameter $\lambda>0$ the basic cell will be identified with the hexagon $\Delta_{\lambda} \equiv \lambda \Delta$, where

$$
\lambda \Delta \equiv\left\{\alpha_{1} P_{1}+\ldots+\alpha_{6} P_{6}: \alpha_{1}+\ldots+\alpha_{6} \leq 1,0 \leq \alpha_{i} \leq 1 \text { for } i=1, \ldots, 6\right\}
$$

and

$$
\begin{gathered}
P_{1} \equiv(1,0,0), P_{2} \equiv(0.5,-\sqrt{3} / 2,0), P_{3} \equiv(-0.5,-\sqrt{3} / 2,0), \\
P_{4} \equiv(-1,0,0), P_{5} \equiv(-0.5, \sqrt{3} / 2,0), P_{6} \equiv(0.5, \sqrt{3} / 2,0)
\end{gathered}
$$

are apexes of basic cell.

Every hexagonal cell $\Delta_{\lambda}(x) \equiv \lambda \Delta(x)$ will be assumed to be obtained as a result $\lambda \Delta(x)=x+\lambda \Delta$ of a translation of basic cell $\Delta_{\lambda} \equiv \lambda \Delta$ from the origin $O$ of the Carthesian coordinate system to the origin $x=\lambda v_{p} p+\lambda v_{q} q+v_{r} r$ of the cell $\lambda \Delta(x), \quad$ where $\quad v_{p}, v_{q}, v_{r} \in R \quad$ and $\quad p=[1.5,0.5 \sqrt{3}, 0], \quad q=[1.5,-0.5 \sqrt{3}, 0]$, $r=[0,0,1]$.

Moreover, setting $\Lambda_{\lambda} \equiv \lambda \Lambda \quad \Lambda \equiv\{k p+l q+\alpha r: \alpha \in R, k, l=\ldots,-1,0,1, \ldots\}$ for the lattice of basic cell origins, in the coverage $\{\lambda \Delta(x)\}_{x \in R^{3}}$ of $R^{3}$ we have introduced the subcover of $\{\lambda \Delta(x)\}_{x \in \Lambda}$ which consists of hexagonal cells $\lambda \Delta(x)=\lambda k p+\lambda l q+v r+\lambda \Delta$ geometrically and materially isomorphic with the basic cell $\lambda \Delta$ [12]. In the introduced Carthesian orthogonal coordinate system $O x^{1} x^{2} x^{3}$ plane $O x^{1} x^{2}$ is a biperiodicity plane and $O x^{3}=O z$ is normal to this plane. The positive real $\lambda$ being a side length of basic cell and, as it has been already mentioned above, it will be referred to as a microstructural parameter provided that it is small when compared with the characteristic length dimension of the region occupied by the conductor in the reference configuration. Hence, $\Delta$ is a regular hexagon with the side length equal to 1 .

Considerations will be restricted to the special kind of the basic cell $\lambda \Delta$ which is divided into three material rhombus parts $\lambda \diamond_{1}, \lambda \Delta_{2}, \lambda \Delta_{3}$, cf. Figure 2, such that we deal with the basic cell with the rotational symmetry. It means that the material structure of every rhombus part can be obtained from the material structure of the other rhombus as a certain rotation over an angle $2 \pi / 3$ with the origin of the introduced Carthesian coordinate system as a center of the rotation. 


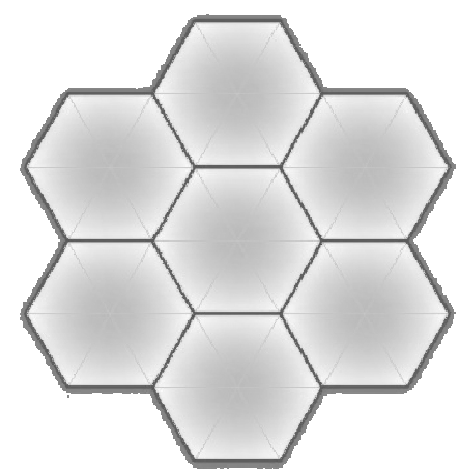

Fig. 1. The conductor with hexagonal-type material structure

Thermal properties of each rhombus are described by heat conductivity tensor $K(\cdot)$ and by specific heat $c(\cdot)$. In the considered case the heat conductivity tensor $K(\cdot)$ in each of the three rhombus parts has form

$$
K(\mathbf{x})=\left\{\begin{array}{l}
K \text { DLA } \mathbf{x}=(x, z) \in \diamond_{I} \times(0, H) \\
Q_{2 \pi / 3}{ }^{T} K Q_{2 \pi / 3} \text { for } \mathbf{x}=(x, z) \in \diamond_{I I} \times(0, H) \\
Q_{2 \pi / 3} K Q_{2 \pi / 3}{ }^{T} \text { for } \mathbf{x}=(x, z) \in \diamond_{I I I} \times(0, H)
\end{array}\right.
$$

Symbol $H$ means the thickness of the conductor layer and $Q_{2 \pi / 3}$ denotes the related rotational matrix

$$
Q_{2 \pi / 3}=\left[\begin{array}{ccc}
\cos \left(\frac{2 \pi}{3}\right) & -\sin \left(\frac{2 \pi}{3}\right) & 0 \\
\sin \left(\frac{2 \pi}{3}\right) & \cos \left(\frac{2 \pi}{3}\right) & 0 \\
0 & 0 & 1
\end{array}\right]=\left[\begin{array}{ccc}
-\frac{1}{2} & -\frac{\sqrt{3}}{2} & 0 \\
\frac{\sqrt{3}}{2} & -\frac{1}{2} & 0 \\
0 & 0 & 1
\end{array}\right]
$$

Moreover, it will be assumed that we deal with the special kind of anisotropic conductor with the conductivity matrix $K(\cdot)$ given by:

$$
K=\left[\begin{array}{ccc}
k_{11} & 0 & 0 \\
0 & k_{22} & 0 \\
0 & 0 & k_{33}
\end{array}\right]
$$

It means that directions of principal axes of the conductivity tensor are congruent with the axes of the spherical coordinate system. For the purpose of further 
consideration there is assumed a jump discontinuous of the heat conductivity matrix on interfaces between three rhombus sections which are treated as perfectly bonded $[13,14]$.

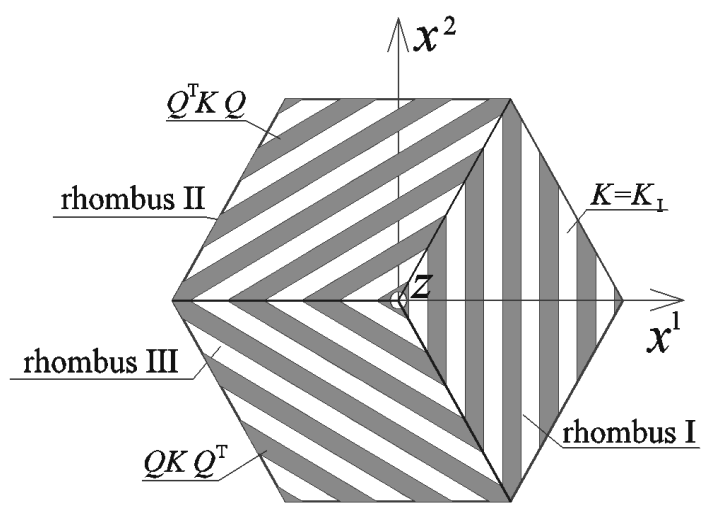

Fig. 2. Basic hexagonal cell

\section{Boundary effect equation}

To formulate the tolerance effect equation we shall use the tolerance modeling technique to the parabolic heat transfer equation with periodic coefficients

$$
c \dot{\theta}-(\nabla+\partial) \cdot[K(\nabla+\partial)] \theta=-b
$$

In the above equation symbol $b=b(x, z)$ means the heat sources field and $\theta=\theta(x, z, t)$ denotes the temperature field. An overdot denotes the time derivative. Moreover, $\partial=\left(0,0, \partial / \partial x^{3}\right)$ and $\nabla=\left(\partial / \partial x^{1}, \partial / \partial x^{2}, 0\right)$. The well-known tolerance averaging technique applied to the equation (4) leads to the model equations:

$$
\begin{gathered}
\langle c\rangle \dot{u}-(\nabla+\partial)^{T}(\langle K\rangle(\nabla+\partial) u-[K] \bar{\psi})=\langle b\rangle \\
\lambda^{2}\left\langle g^{a} c g^{b}\right\rangle \dot{\bar{\psi}}-\lambda^{2}\left(k_{33}\right) \partial_{3} \partial_{3} \bar{\psi}-\lambda(\kappa) \partial_{3} \bar{\psi}+\{K\} \bar{\psi}+[K]^{T} \nabla u=\lambda \beta
\end{gathered}
$$

with basic unknowns $u=u(x, z, t)$ and $\bar{\psi}=\bar{\psi}(x, z, t)$, cf. [1-4]. The first basic unknown $u=u(x, z, t)$ is the averaged temperature field defined by $u(x, z, t)=\langle\theta\rangle(x, z, t)$ for integral averaging operation over the hexagonal cell $\Delta(x)$ :

$$
\langle f\rangle(x)=\frac{1}{\operatorname{area}(\Delta)} \int_{\Delta} f(x+y) d y
$$


The second basic unknown $\bar{\psi}=\bar{\psi}(x, z, t)$ is $N$-tuple $\bar{\psi}=\left[\psi^{1}, \ldots, \psi^{n}\right]^{T}$ in which $n$ is the number of shape functions and where $\psi^{a}=\psi^{a}(x, z, t), a=1, \ldots, n$, are referred to as fluctuation amplitudes and is interrelated with temperature field $\theta=\theta(x, z, t)$ and averaged temperature field $u=u(x, z, t)$ by the micro-macro hypothesis which postulates that the temperature field can be approximated by:

$$
\theta=u+h^{a} \psi^{a}
$$

Here and in the sequel small Latin sub- and super-scripts $a, b, c, \ldots$ run over $1, \ldots, n$ and $h^{a}=\lambda g^{a}(x), a=1, \ldots, n$, is the sequence of shape functions which should be postulated in every tolerance modeling problem. The matrix coefficients in (5) are given by:

$$
\begin{aligned}
& {[K]=\left[\left\langle\operatorname{Kradg}^{a}\right\rangle\right]} \\
& {[K]^{T}=\left[\left\langle\operatorname{grad}^{T} g^{a} K\right\rangle\right]} \\
& \{K\}=\left[\left\langle\operatorname{grad}^{T} g^{a} \operatorname{Kgradg}^{b}\right\rangle\right] \\
& \left(k_{33}\right)=\left[\left\langle g^{a} k_{33} g^{b}\right\rangle\right] \\
& (\kappa)=\left[2\left\langle\kappa^{T} g^{a} \operatorname{gradg}^{b}\right\rangle\right] \\
& \beta=\left[\left\langle g^{a} b\right\rangle\right]
\end{aligned}
$$

The second equation from (5) can be treated as a system of ordinary differential equations with respect to the unknown functions $\psi^{1}, \psi^{2}, \psi^{3}$ and the boundary effect equation will be identified in this paper (by definition) as the homogeneous part of this system, i.e.

$$
\lambda^{2}\left(k_{33}\right) \partial_{3} \partial_{3} \bar{\psi}-\{K\} \bar{\psi}=0
$$

The choice of the form and the number of shape functions $h^{a}=\lambda g^{a}(x)$, $a=1, \ldots, n$, is in fact the crucial part of the procedures which in many papers are referred to as tolerance modeling. For example, in the tolerance modeling of uniperiodic two-constituent laminates the saw-like function seems to be sufficient to obtain models that yield acceptable approximate description of various physical problems.

In the framework of the realization of the tolerance modelling of heat transfer behaviors in the hexagonal-type rigid conductors, the system of three shape functions $\lambda g^{1}, \lambda g^{2}, \lambda g^{3}$ will be taken into account. Hence the tolerance averaging modeling procedure will be realized for $n=3$ and in the framework of the investigated tolerance model the averaged heat conduction will be described by four basic 
unknowns $u, \psi^{1}, \psi^{2}, \psi^{3}$ consisting of averaged temperature field $u$ and three fields of fluctuation amplitudes $\psi^{1}, \psi^{2}, \psi^{3}$.

\section{Modeling procedure}

The boundary effect equation (9) will be more widely evaluated after introducing shape functions $g^{1}, g^{2}, g^{3}$ interrelated by conditions:

$$
\begin{gathered}
g^{1} \equiv g_{\text {peaks }}\left(\lambda^{-1} x\right) \\
g^{2} \equiv g_{\text {peaks }}\left(\lambda^{-1} \operatorname{rot}_{Q}(x)\right) \\
g^{3} \equiv g_{\text {peaks }}\left(\lambda^{-1} \operatorname{rot}_{Q} \circ \operatorname{rot}_{Q}(x)\right)
\end{gathered}
$$

where $\operatorname{rot}_{\mathrm{Q}}(x)=Q_{2 \pi / 3}\left(x-x_{0}\right)^{T}+x_{0}, x \in R^{2}$ is the rotation over $2 \pi / 3$ in $R^{3}$ with an arbitrary chosen origin $X \in \lambda \Lambda$ of a hexagonal cell as a center of rotation. This means that the shape function system is generated by single function $g_{\text {peaks }}$ which is built with pieces of linear function of variables $x_{1}, x_{2}$ and has been presented in Figure 3. It will be referred to as a generating function. Under micro-macro hypothesis (7) conditions (10) can be interpreted as a certain realization of hypothesis that in the first approximation the difference $\theta-u$ between the temperature field $\theta$ and averaged temperature field $u$ is, for every three directions intersected over the angles $2 \pi / 3$, based on the same temperature shape determined here, under formulas (10), by generating function $g_{\text {peaks }}$.

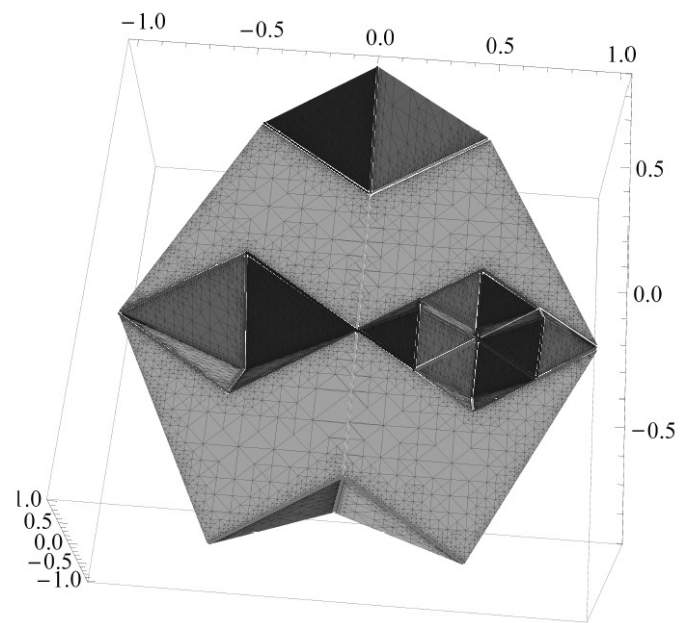

Fig. 3. Function $g_{\text {peaks }}$ 
To define a generating function $g_{\text {peaks }}$ let:

$$
\begin{aligned}
& \gamma_{1}(x)=\max \left\{0, \min \left\{\alpha(x), \alpha\left(\operatorname{rot}_{\pi / 3}(x)\right),\right.\right. \\
& \left.\alpha\left(\operatorname{rot}_{-\pi / 3}(x)\right), \alpha\left(\operatorname{rot}_{2 \pi / 3}(x)\right), \alpha\left(\operatorname{rot}_{-2 \pi / 3}(x)\right)\right\}
\end{aligned}
$$

where $\alpha(x)=2\left[-\frac{1}{\sqrt{3}}\left(x_{1}-\frac{\sqrt{3}}{2}\right)-x_{2}\right]$ and:

$$
\begin{aligned}
& \gamma_{s+1}(x)=\max \left\{\gamma_{k}\left(2\left(x-\left(\frac{\sqrt{3}}{4}, 0\right)\right)\right),\right. \\
& \left.\gamma_{s}\left(2\left(x-\left(-\frac{\sqrt{3}}{4}, 0\right)\right)\right)\right\}-\max \left\{\gamma_{k}\left(2\left(x-\left(0 . \frac{1}{4}\right)\right)\right), \gamma_{k}\left(2\left(x-\left(0,-\frac{1}{4}\right)\right)\right)\right\}
\end{aligned}
$$

for any integer $s>0$. Finally let:

$$
\bar{\gamma}_{s}\left(\frac{2 \sqrt{3}}{3} x\right)=\left\{\begin{array}{c}
\gamma_{s}\left(x-\left(\frac{1}{2}, 0\right)\right) \quad \text { for } \quad x \in \diamond_{I} \\
\gamma_{1}\left(x-\left(0,-\frac{1}{2}\right)\right)-\max \left\{\gamma_{1}\left(x-\left(0, \frac{\sqrt{3}}{2}\right)\right), \gamma_{1}\left(x-\left(0,-\frac{\sqrt{3}}{2}\right)\right)\right\} \\
\text { for } x \in \diamond_{I I} \cup \diamond_{I I I}
\end{array}\right.
$$

and $\bar{\gamma}(x+k p+l q)=\bar{\gamma}(x)$ for $x \in \Delta$ and arbitrary integers $k, l$. Note that $\left\langle\nabla \delta_{s}\right\rangle=0$ as well as $\sup _{x \in \Delta}\left|\delta_{s}(x)\right|=2^{-s}$ for any positive integer $k$ and hence $\lim _{s \rightarrow+\infty} \sup _{x \in \Delta}\left|\delta_{s}(x)\right|=0$. Bearing in mind that for just defined function $\bar{\gamma}_{s}(x)$ (as well as for every piecewise linear function) an infinitely closed to $\bar{\gamma}_{s}(x)$ in $H_{p e r}^{1}(R)$ tolerance shape function $\lambda \delta_{s}(x)$ exists and satisfies conditions $\left|\bar{\gamma}_{s}(x)-\delta_{s}(x)\right|<\frac{1}{s},\left\|\nabla \bar{\gamma}_{s}(x)-\nabla \delta_{s}(x)\right\|<\frac{1}{s}$. Finally, independently for any positive integer $s$ we define $g_{\text {peaks }}=\delta_{s}$. For $s=2$ generating function $g_{\text {peaks }}=\delta_{2}$ is illustrated onto Figure 3. Limit passage $s \rightarrow+\infty$ applied to model equations (5) as well to the boundary effect equation (9) not remain unchanged all coefficients used coefficients. Under this limit passage coefficients $\{K\}$ and $\left(k_{33}\right)$ with $s \rightarrow+\infty$ take forms:

$$
\begin{aligned}
& \{K\}=\{k\} I, \quad\{k\} \equiv \frac{1}{6}\left(k_{11}+k_{22}\right) \\
& \left(k_{33}\right)=(k) I, \quad(k) \equiv 2 \cdot 0.06 \cdot k_{33}
\end{aligned}
$$


where $I$ denotes the unit $3 \times 3$ matrix. As the most important fact it will be emphasized that for introduced shape function it is possible to satisfy the continuity condition for the component of the tolerance heat flux vector, normal to planes dividing regions occupied by different materials, cf. [15]. That is why the fluctuation amplitudes $\psi_{1}, \psi_{2}, \psi_{3}$ can be treated as temperature fluctuation amplitudes. Moreover, under (14), boundary effect equation (6) separates for three second order ordinary differential equations with the same coefficients which depend on two material parameters $k_{11}+k_{22}$ and $k_{33}$.

\section{Illustrative problem}

To illustrate theoretical considerations in the previous sections, let us consider the stationary heat transfer problem for layer $\Omega \equiv \Xi \times(0, H)$, layer $\Xi \subset R^{2}$, layer $(0, H) \subset R$, occupied by the hexagonal-type periodic conductor, cf. Figure 4 , and assume boundary conditions:

$$
\begin{gathered}
\theta(x, 0)=\theta_{0} \equiv u_{0}+\lambda g^{a} \psi_{0}^{a}, \quad \theta(x, 0)=\theta_{H} \equiv u_{H}+\lambda g^{a} \psi_{H}^{a}, \\
u_{0} \neq u_{H}
\end{gathered}
$$

for tolerance model equations:

$$
\begin{gathered}
(\nabla+\partial)^{T}(\langle K\rangle(\nabla+\partial) u+[K] \bar{\psi})=-\langle b\rangle \\
\lambda^{2}\left(k_{33}\right) \partial_{3} \partial_{3} \bar{\psi}-\{K\} \bar{\psi}-[K]^{T} \nabla u=-\lambda \beta
\end{gathered}
$$

and for given boundary constant values $u_{0}, u_{H}$ and $\psi_{0}^{a}, \psi_{H}^{a}, a=1,2,3$, $\bar{\psi} \equiv\left(\psi^{1}, \psi^{2}, \psi^{3}\right)$.

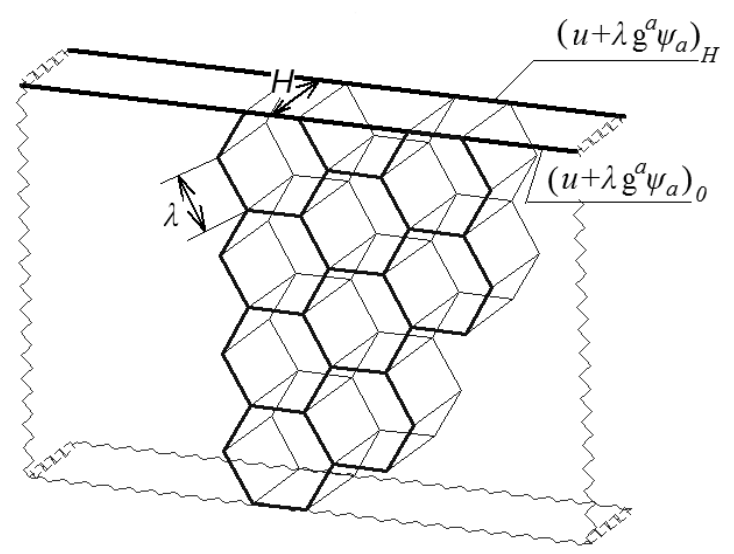

Fig. 4. Hexagonally periodic conductor 
To avoid unnecessary misunderstandings, we shall assume that region $\Xi \subset R^{2}$ is a sum of finite (but large) number of hexagonal cells, i.e.

$$
\Xi=\bigcup\left\{k \mathrm{p}+l \mathrm{q}+\Delta: \quad k=k_{1}, k_{1}+1, \ldots, k_{2}-1, k_{2}, \quad l=l_{1}, l_{1}+1, \ldots, l_{2}-1, l_{2}\right\}
$$

for certain integers $k_{1}, k_{2}, l_{1}, l_{2}$ and for $\mathrm{p} \equiv[1.5,0.5 \sqrt{3}], \mathrm{q} \equiv[1.5,-0.5 \sqrt{3}]$. Moreover, assume that $b=0$.

The solution to the formulated problem is given by

$$
\theta(x, z)=\tilde{u}(z)+\lambda g^{a}(x) \tilde{\psi}^{a}(z)
$$

for

$$
\tilde{u}(z)=\frac{u_{H}-u_{0}}{H} z+u_{0}
$$

and for $\bar{\psi}=\bar{\psi}(z)$ satisfying boundary effect equation (9). The form of the solution $\bar{\psi}=\bar{\psi}(z)$ of the boundary effect equation (9) will be discussed in the next section.

\section{Solution to the boundary effect equation}

The boundary effect equation (9) is a matrix equation which consists of three independent equations for unknowns $\psi^{1}, \psi^{2}, \psi^{3}$. These equations have the same coefficients and that is why fluctuation amplitudes a determined by the related boundary conditions imposed on these equations. Hence, we reduce boundary values for amplitudes $\psi^{1}, \psi^{2}, \psi^{3}$ to the simplest form:

$$
\bar{\psi}_{0} \equiv\left[\psi_{0}^{1}, \psi_{0}^{2}, \psi_{0}^{3}\right]=\psi_{0}[1,1,1]^{T}, \quad \bar{\psi}_{H} \equiv\left[\psi_{H}^{1}, \psi_{H}^{2}, \psi_{H}^{3}\right]=\psi_{H}[1,1,1]^{T}
$$

under which amplitudes $\psi^{1}, \psi^{2}, \psi^{3}$ are identical functions of variable $x_{3}$, namely $\psi\left(x_{3}\right)$. Then there can be used solution to the similar simpler problem formulated and investigated in [16].

$$
\begin{aligned}
& \psi=\frac{e^{-\frac{1}{\lambda} \sqrt{\left(k_{33}\right)^{-1}\{k\}} x_{3}}}{e^{2 \frac{H}{\lambda} \sqrt{\left(k_{33}\right)^{-1}\{k\}}}-I}\left(e^{2 \frac{H}{\lambda} \sqrt{\left(k_{33}\right)^{-1}\{k\}}} \bar{\psi}_{0}-e^{\frac{H}{\lambda} \sqrt{\left(k_{33}\right)^{-1}\{k\}}} \psi_{H}\right)+ \\
& +\frac{e^{\frac{1}{\lambda} \sqrt{\left(k_{33}\right)^{-1}\{k\}} x_{3}}}{e^{2 \frac{H}{\lambda} \sqrt{\left(k_{33}\right)^{-1}\{k\}}}-I}\left(-\bar{\psi}_{0}+e^{\frac{H}{\lambda} \sqrt{\left(k_{33}\right)^{-1}\{k\}}} \psi_{H}\right)
\end{aligned}
$$

In the above solution Lapunov exponent $\exp \left(\frac{\lambda}{H} \sqrt{\left(K_{33}\right)^{-1}\{K\}}\right.$ has been used, cf. [17]. Moreover, fractions of two martices mean the commutative multiplication 
of the numerator and the inversion of denominator. Introducing new designations for $\lambda / H$ and bearing in mind that matrix exponents used in (20) are proportional to the unit matrix we get:

$$
\begin{aligned}
& \psi=\frac{e^{-\frac{1}{\lambda} \sqrt{\chi} x_{3}}}{e^{2 \frac{1}{\eta} \sqrt{\chi}}-1}\left(e^{\frac{2}{\eta} \sqrt{\chi}} \psi_{0}-e^{\frac{1}{\eta} \sqrt{\chi}} \psi_{H}\right)+ \\
& +\frac{e^{\frac{1}{\lambda} \sqrt{\chi} x_{3}}}{e^{2 \frac{1}{\eta} \sqrt{\chi}}-1}\left(-\psi_{0}+e^{\frac{1}{\eta} \sqrt{\chi}} \psi_{H}\right)
\end{aligned}
$$

where:

$$
\begin{gathered}
\eta=\frac{\lambda}{H} \\
\chi I=\left(k_{33}\right)^{-1}\{K\}
\end{gathered}
$$

In the considered case coefficient $\chi$ takes the form:

$$
\chi=\frac{\frac{1}{6}\left(k_{11}+k_{22}\right)}{2 \cdot 0.06 \cdot k_{33}}
$$

The largest boundary effect appears at points of the conductor, in which values of functions $g^{1}, g^{2}, g^{3}$ are highest. Related graphs have been presented for the following values:

$$
\begin{array}{ll}
u_{H}=283 \mathrm{~K} & \psi_{H}=20 \mathrm{~K} \\
u_{0}=273 \mathrm{~K} & \psi_{0}=40 \mathrm{~K} \\
H=0.2 \mathrm{~m} & \lambda=0.04 \mathrm{~m}
\end{array}
$$

Four examples of material structure have been presented. In first three examples the components of heat conductivity tensor $K$ have form:

$$
\begin{aligned}
k_{11} & =\frac{1}{\frac{s_{1}}{k^{I}}+\frac{s_{2}}{k^{I I}}} \\
k_{22}= & k_{33}=s_{1} k^{I}+s_{2} k^{I I}
\end{aligned}
$$

which corresponds to elements of the well-known tolerance effective conductivity matrix of two-layer laminate [2]. In the above formulas heat conductivities of layers are denoted by $k^{I}, k^{I I}$, respectively, and the related thickness of layers by 
$l_{1}, l_{2}$. Hence, $s_{1}=l_{1} /\left(l_{1}+l_{2}\right), s_{2}=l_{2} /\left(l_{1}+l_{2}\right)$ are related material fractions. In fourth example the heat conductivity tensor $K$ is a diagonal matrix with different conductivity properties in every of three directions designated by axes of coordinate system. Conductor of the first rhombus in above examples is taken as follows:

Example 1. $k^{I}=58, k^{I I}=0.025 \mathrm{~W} / \mathrm{m} \cdot \mathrm{K}$ and $l_{1}=0.005, l_{2}=0.005 \mathrm{~m}$,

Example 2. $k^{I}=58, k^{I I}=0.025 \mathrm{~W} / \mathrm{m} \cdot \mathrm{K}$ and $l_{1}=0.008, l_{2}=0.002 \mathrm{~m}$,

Example 3. $k^{I}=0.045, k^{I I}=0.025 \mathrm{~W} / \mathrm{m} \cdot \mathrm{K}$ and $l_{1}=0.01, l_{2}=0.01 \mathrm{~m}$,

Example 4. $k_{11}=58, k_{22}=1, k_{33}=0.025 \mathrm{~W} / \mathrm{m} \cdot \mathrm{K}$.

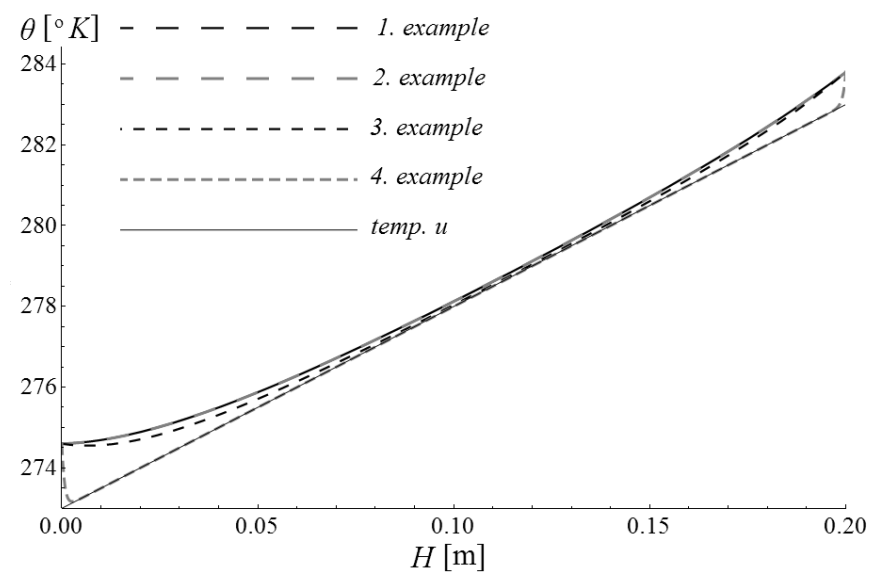

Fig. 5. Solution to the boundary effect issue

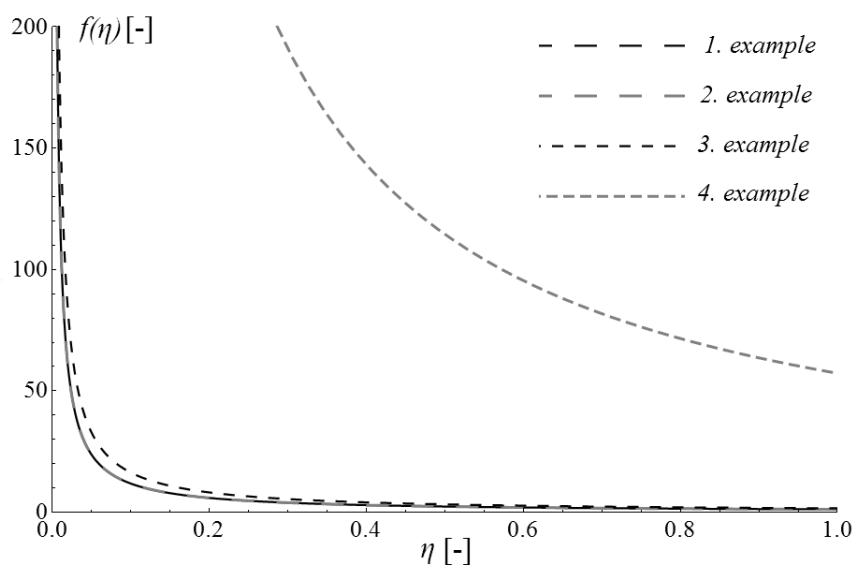

Fig. 6. The interrelation between the intensity of the boundary effect behavior and nondimensional microstructure parameter $\eta$ 
Quotient $\sqrt{\chi} / \eta$ can be treated as a certain measure of the intensity of boundary effect behavior and will be referred to as the boundary effect intensity parameter. The intensity of boundary effect behavior is the highest when the layer of suppression of fluctuation amplitudes is the thinnest. The above graph illustrates the interrelation between the intensity of boundary effect behavior $f(\eta)=\sqrt{\chi} / \eta$ and nondimentional microstructure parameter $\eta$ for four presented examples.

\section{Final remarks}

The interpretation of the solution illustrated in Figure 5 is related to results presented in Figure 6. The intensity of boundary effect grows with decrease of nondimensional microstructure parameter $\eta$. The boundary effect is stronger for the smaller cell and larger width of the dividing wall.

The lines presented onto both Figures yield conclusions:

1. The most intense of boundary effect appears for small dimension of cell $\lambda$ and small value of $\eta$.

2. The highest intensity of boundary effect behavior is observed for the fourth example when the components of heat conductivity tensor $K$ have very different values.

3. If rhombus parts of the considered conductor are made of two-layer laminates then the most intense boundary effect appears for two materials with very similar heat properties, which is the opposite results obtained in the fourth example. It must be also emphasized that for the first two examples the results are the same despite the fact that thickness of two layers in both examples are different. The reason for this is adaptation of the tolerance effective conductivity matrix of two-layer laminate to calculations in examples 1,2 and 3.

4. The intensity of boundary effect behavior is strictly connected with the matrix indicator $\frac{H}{\lambda} \sqrt{\left(k_{33}\right)^{-1}\{K\}}$ of the Lapunov exponent $\exp \left(\frac{H}{\lambda} \sqrt{\left(k_{33}\right)^{-1}\{K\}}\right)$. Hence, this intensity is independent of the form of boundary conditions (14).

It must be emphasized that, as usual in tolerance modeling, results obtained in this paper have only a qualitative character. The obtained solution of boundary effect equation for the considered hexagonal type rigid conductor leads to the conclusion that the intensity of boundary effect depends on parameters $k_{11}+k_{22}$ and $k_{33}$ placed in the indicator $\frac{H}{\lambda} \sqrt{\left(k_{33}\right)^{-1}\{K\}}$ of the Lapunov exponent $\exp \left(\frac{H}{\lambda} \sqrt{\left(k_{33}\right)^{-1}\{K\}}\right)$, as it has been mentioned in the $4^{\text {th }}$ conclusion. 


\section{References}

[1] Jedrysiak J., Termomechanika laminatów, płyt i powłok o funkcyjnej gradacji własności, Lodz Technical University Press, Lodz 2010.

[2] Woźniak C., Wierzbicki E., Averaging Techniques in Thermomechanics of Composite Solids, Częstochowa University of Techn. Press, Częstochowa 2000.

[3] Woźniak C. (ed.), Developments in Mathematical Modeling and Analysis of Microstructured Media, Silesian Techn. University Press, Gliwice 2010.

[4] Woźniak C. (ed.), Thermomechanics of Microheterogeneous Solids and Structures. Tolerance Averaging Approach, Lodz Technical University Press, Lodz 2009.

[5] Kula D., Mazewska M, Wierzbicki E., Some remarks on the tolerance averaging of heat conduction In chessboard palisade-type periodic composites, Scientific Review, Engineering and Enviromental Sciences 2012, 21(3), 57, 131-140.

[6] Mazewska M., Wierzbicki E., Modelowanie tolerancyjne przewodnictwa ciepła w kompozytach o strukturze dwukierunkowo-periodycznej, Acta Scientarum Polonarum, Architectura 2013, 12(1), 3-17.

[7] Michalak B., Termomechanika ciał z pewną niejednorodną mikrostrukturą: technika tolerancyjnej aproksymacji, Lodz Technical University Press, Lodz 2010.

[8] Nagórko W., Wybrane metody modelowania płyt niejednorodnych, Publications of Warsaw University of Life Sciences - SGGW, Warsaw 2008.

[9] Cielecka I., Jędrysiak J., A non-asymptotic model of dynamics of honeycomb lattice-type plates, J. Sound and Vibration 2006, 296, 130-149.

[10] Nagórko W., Wągrowska M., A contribution to modelling of composite solids, J. Theor. Appl. Mech. 2002, 40, 149-158.

[11] Wierzbicki E., Woźniak C., On the dynamic behaviour of honeycomb based composite solids, Acta Mechanica 2000, 141, 161-172.

[12] Truesdell C., A First Course in Rational Continuum Mechanics, The John Hopkins University, Baltimore, Maryland 1972.

[13] Sideman S., Moalem-Maron D., Direct Contact Condensation, Advances in Heat Transfer, Academic Press, New York 1982, 228-276.

[14] Vutz N., Angrist S.W., Thermal Contact Resistance of Anisotropic Materials, J. Heat Transfer 1970, 92(1), 17-20.

[15] Mazewska M., Tolerance Modeling of Boundary Effect Behavior in Hexagonal-type Composites, Doctoral dissertation, Warsaw University of Life Sciences, Warsaw 2013.

[16] Woźniak M., Wierzbicki E., Woźniak C., A macroscopic model of the diffusion and heat transfer processes in a periodically micro-stratified solid layer, Acta Mechanica 2002, 157, 175-185.

[17] Lai-Sang Young, Mathematical theory of Lyapunov exponents, Journal of Physics A: Mathematical and Theoretical 2013, 46, 254001. 